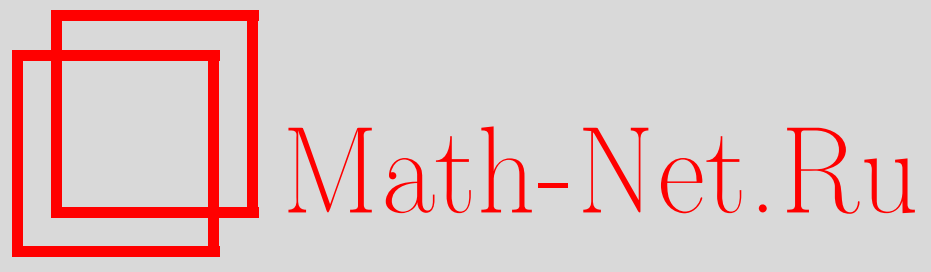

О. И. Мохов, Системы интегралов в инволюции и уравнения ассоциативности, УМH, 2006, том 61, выпуск $3,175-176$

DOI: https://doi.org/10.4213/rm1755

Использование Общероссийского математического портала Math-Net.Ru подразумевает, что вы прочитали и согласны с пользовательским соглашением http://www . mathnet.ru/rus/agreement

Параметры загрузки:

IP : 54.196 .121 .252

26 апреля 2023 г., 14:59:37

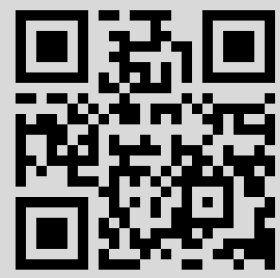




\section{Системы интегралов в инволюции и уравнения ассоциативности}

\section{О. И. Мохов}

В данной работе, в частности, доказано, что уравнения ассоциативности двумерных топологических квантовых теорий поля (см. [1]-[3]) эквивалентны условию, что все функционалы, плотности которых являются частными производными первого порядка некоторой функции $F\left(u^{1}, \ldots, u^{N}\right)$ (“потенциала"), находятся в инволюции относительно постоянной однородной скобки Пуассона первого порядка. Действительно, рассмотрим произвольную постоянную однородную скобку Пуассона первого порядка, т.е. теоретико-полевую скобку Пуассона

$$
\left\{u^{i}(x), u^{j}(y)\right\}_{1}=\eta^{i j} \delta^{\prime}(x-y),
$$

где $\eta^{i j}$ - произвольная симметричная постоянная матрица, $\eta^{i j}=\eta^{j i}, \eta^{i j}=$ const; $u=\left(u^{1}, \ldots, u^{N}\right)$ - фиксированные локальные координаты в некоторой рассматриваемой односвязной области $U ; u^{i}(x), i=1, \ldots, N,-$ функции одной переменной ( $N$ скалярных полей на одномерном многообразии), $u(x)=\left(u^{1}(x), \ldots, u^{N}(x)\right)-N$-компонентное скалярное поле со значениями в области $U$. Пусть $F\left(u^{1}, \ldots, u^{N}\right)$ - произвольная гладкая функция $N$ переменных, определенная в области $U$. Рассмотрим на пространстве полей $u(x) \quad N$ функционалов $\mathscr{F}_{i}, i=1, \ldots, N$, плотности которых являются частными производными первого порядка функции $F\left(u^{1}, \ldots, u^{N}\right)$ :

$$
\mathscr{F}_{i}=\int \frac{\partial F}{\partial u^{i}}\left(u^{1}(x), \ldots, u^{N}(x)\right) d x, \quad i=1, \ldots, N .
$$

Теорема 1. Функииональ $\mathscr{F}_{i}, i=1, \ldots, N$, находятся в инволюции относительно постоянной однородной скобки Пуассона первого порлдка (1), m.е. $\left\{\mathscr{F}_{i}, \mathscr{F}_{j}\right\}_{1}=0$, $i, j=1, \ldots, N$, тогда и толъко тогда, когда функиия $F\left(u^{1}, \ldots, u^{N}\right)$ удовлетворяет уравнениям ассоциативности двумерных топологических квантовых теорий поля:

$$
\frac{\partial^{3} F}{\partial u^{k} \partial u^{m} \partial u^{i}} \eta^{i j} \frac{\partial^{3} F}{\partial u^{j} \partial u^{n} \partial u^{l}}=\frac{\partial^{3} F}{\partial u^{l} \partial u^{m} \partial u^{i}} \eta^{i j} \frac{\partial^{3} F}{\partial u^{j} \partial u^{n} \partial u^{k}} .
$$

Матрица $\eta^{i j}$ не предполагается здесь невырожденной. Если для $F\left(u^{1}, \ldots, u^{N}\right)$ выполнены уравнения (3), то для любых значений координат $u^{1}, \ldots, u^{N}$ в области $U$ $N$-мерная алгебра $\mathscr{A}$ с базисом $\left(e_{1}, \ldots, e_{N}\right)$ и умножением $e_{i} \cdot e_{j}=c_{i j}^{k}\left(u^{1}, \ldots, u^{N}\right) e_{k}$, где $c_{i j}^{k}\left(u^{1}, \ldots, u^{N}\right)=\eta^{k s} \partial^{3} F /\left(\partial u^{s} \partial u^{i} \partial u^{j}\right)$, является ассоциативной. В топологических теориях поля матрица $\eta^{i j}$ является невырожденной (она играет роль плоской контравариантной метрики) и в этом случае алгебра $\mathscr{A}$ является ассоциативной тогда и только тогда, когда выполнены уравнения (3), называемые уравнениями ассоциативности или уравнениями Виттена-Дейкграфа-Верлинде-Верлинде и Дубровина [1]-[3]. Матрица $\eta_{i j}$ (ковариантная метрика), обратная к невырожденной матрице $\eta^{i j}$, $\eta_{i s} \eta^{s j}=\delta_{i}^{j}$, задает невырожденную инвариантную симметричную билинейную форму $\left\langle e_{i}, e_{j}\right\rangle=\eta_{i j}$ на алгебре $\mathscr{A}:\left\langle e_{i} \cdot e_{j}, e_{k}\right\rangle=\left\langle e_{i}, e_{j} \cdot e_{k}\right\rangle$. Переопределенная нелинейная система уравнений ассоциативности (3) является совместной и интегрируемой методом обратной задачи. Большое количество примеров точных решений уравнений ассоциативности (3) см. в [3]. Пусть матрица $\eta^{i j}$ является невырожденной, $\operatorname{det} \eta^{i j} \neq 0$. В работе автора [4] доказано, что если функция $F\left(u^{1}, \ldots, u^{N}\right)$ является решением уравнений ассоциативности (3), то оператор

$$
M^{i j}=\eta^{m n} \eta^{i p} \eta^{j r} \frac{\partial^{3} F}{\partial u^{p} \partial u^{m} \partial u^{k}} u_{x}^{k}\left(\frac{d}{d x}\right)^{-1} \circ \frac{\partial^{3} F}{\partial u^{r} \partial u^{n} \partial u^{s}} u_{x}^{s}
$$

Работа выполнена при финансовой поддержке Фонда содействия отечественной науке, Российского фонда фундаментальных исследований (гранты № 03-01-00782 и № 05-01-00170) и Программы поддержки ведущих научных школ (грант НШ-4182.2006.1). 
является гамильтоновым.

Теорема 2. Если функиия $F\left(u^{1}, \ldots, u^{N}\right)$ удовлетворяет уравнениям ассоциативности (3), то функциональ $\mathscr{F}_{i}, i=1, \ldots, N$, находятся в инволюиии относительно скобки Пуассона, задаваемой гамильтоновым оператором (4), т.е. $\left\{\mathscr{F}_{i}, \mathscr{F}_{j}\right\}_{2}=0$, $i, j=1, \ldots, N$, где

$$
\left\{u^{i}(x), u^{j}(y)\right\}_{2}=\eta^{m n} \eta^{i p} \eta^{j r} \frac{\partial^{3} F}{\partial u^{p} \partial u^{m} \partial u^{k}} u_{x}^{k}\left(\frac{d}{d x}\right)^{-1}\left(\frac{\partial^{3} F}{\partial u^{r} \partial u^{n} \partial u^{s}} u_{x}^{s} \delta(x-y)\right) .
$$

В работе автора [4] доказано, что скобки Пуассона $\{\cdot, \cdot\}_{1}$ и $\{\cdot, \cdot\}_{2}$ согласованы и образуют пучок $\lambda_{1}\{\cdot, \cdot\}_{1}+\lambda_{2}\{\cdot, \cdot\}_{2}$ скобок Пуассона, где $\lambda_{1}$ и $\lambda_{2}-$ произвольные константы, и для любых констант $\lambda_{1}$ и $\lambda_{2}$ скобка $\{\cdot, \cdot\}_{\lambda_{1}, \lambda_{2}}=\lambda_{1}\{\cdot, \cdot\}_{1}+\lambda_{2}\{\cdot, \cdot\}_{2}$ является скобкой Пуассона.

СледСтвиЕ 1. Если функиия $F\left(u^{1}, \ldots, u^{N}\right)$ удовлетворяет уравнениям ассоциативности (3), то функциональ $\mathscr{F}_{i}, i=1, \ldots, N$, находятся в инволюции относительно пучка скобок Пуассона $\{\cdot, \cdot\}_{\lambda_{1}, \lambda_{2}}=\lambda_{1}\{\cdot, \cdot\}_{1}+\lambda_{2}\{\cdot, \cdot\}_{2}$, где $\lambda_{1}$ u $\lambda_{2}-$ произвольные константы.

Рассмотрим также на пространстве полей $u(x)$ функционал $\mathscr{F}$, плотностью которого является сам "потенциал" $F\left(u^{1}(x), \ldots, u^{N}(x)\right): \mathscr{F}=\int F\left(u^{1}(x), \ldots, u^{N}(x)\right) d x$.

Теорема 3. Функционал $\mathscr{F}$ и функционалы $\mathscr{F}_{i}, i=1, \ldots, N$, находятся в инволюции относительно постоянной однородной скобки Пуассона первого порядка (1) тогда и толъко тогда, когда функиия $F\left(u^{1}, \ldots, u^{N}\right)$ удовлетворяет уравнениям

$$
\frac{\partial^{2} F}{\partial u^{k} \partial u^{i}} \eta^{i j} \frac{\partial^{3} F}{\partial u^{j} \partial u^{n} \partial u^{l}}=\frac{\partial^{2} F}{\partial u^{l} \partial u^{i}} \eta^{i j} \frac{\partial^{3} F}{\partial u^{j} \partial u^{n} \partial u^{k}} .
$$

Уравнения (6) возникли в другом контексте в работах автора [5]-[7] и играют важную роль в теории согласованных скобок Пуассона гидродинамического типа, теории уравнений ассоциативности и теории фробениусовых многообразий.

Теорема 4. Если функиия $F\left(u^{1}, \ldots, u^{N}\right)$ удовлетворяет уравнениям (6), то функционал $\mathscr{F}$ и функциональ $\mathscr{F}_{i}, i=1, \ldots, N$, находятся в инволючии относительно скобки Пуассона, задаваемой гамильтоновым оператором (4).

СледСтвиЕ 2. Если функция $F\left(u^{1}, \ldots, u^{N}\right)$ удовлетворяет уравнениям (6), то функционал $\mathscr{F}$ и функциональ $\mathscr{F}_{i}, i=1, \ldots, N$, находятся в инволюиии относительно пучка скобок Пуассона $\{\cdot, \cdot\}_{\lambda_{1}, \lambda_{2}}=\lambda_{1}\{\cdot, \cdot\}_{1}+\lambda_{2}\{\cdot, \cdot\}_{2}$, где $\lambda_{1} u \lambda_{2}-$ произвольные константы.

\section{Список литературы}

[1] E. Witten, Surv. Differ. Geom., 1 (1991), 243-310. [2] R. Dijkgraaf, H. Verlinde, E. Verlinde, Nuclear Phys. B, 352:1 (1991), 59-86. [3] B. Dubrovin, Integrable systems and quantum groups (Montecatini Terme, 1993), Lecture Notes in Math., 1620, 1996, 120-348; arXiv: hep-th/ 9407018. [4] О.И. Мохов, Функи. анализ и его прилож., 40:1 (2006), 14-29; arXiv: hep-th/ 0406292. [5] О. И. Мохов, УМН, 53:2 (1998), 153-154. [6] О. I. Mokhov, Rep. Math. Phys., 43:1/2 (1999), 247-256. [7] О. И. Мохов, Солитонь, геометрия, топология - на перекрестках, Труды МИАН, 225, 1999, 284-300.

\section{О. И. Мохов (О. I. Mokhov)}

Центр нелинейных исследований при Институте теоретической физики им. Л. Д. Ландау РАН

E-mail: mokhov@mi.ras.ru, mokhov@landau.ac.ru, mokhov@bk.ru
Представлено С. П. Новиковым Принято редколлегией 02.06.2006 